\title{
Vocalismo y Consonantismo Históricos en el Lenguaje de los Cronistas del Perú.
}

Sy queredes, senores, oyr un buen solaz, ascuchad el rromance, sosegadvos en paz...

Arcipreste de Hita

Libro de Buen Amor - 14 .

Los cronista antiguos del Perú escriben en una prosa que, por lo general, tiene todavía el poder dinamogénico del romance español de le Edad Media. Están insertos dentro de la gran corriente de aquel lenguaje jocundo y móvil que desborda su fuerza plástica y que aun no alcanza a detenerse en las altas formas, complejas y elegantes pero ya cristalizadas, del Renacimiento. Âmérica recibe con él su esencia expresiva y funda' sa. Pradicióneldiomáticar según la sagaz observación de Ricardo Rojas en el frontal de "Eurindia".

Este carácter transformador y progresista del castellano medioeval lo distingue de los demás dialectos que se hablan en la península ibérica. (1). A raíz de la invasión musulmana España queda dividida en dos' grandes secciones, una libre con centro inicial en Oviedo y otra cautiva, cuya sede primitiva es Córdoba. En ambas persiste la lengua hispánica, pero en la primera fermenta y desarrolla al mismo tiempo que la epopeya de la Reconquista. Parece que siguiera a ésta como su necesario instrumento espiritual. El dialecto que prepondera de inmediato es el asturiano, en los siglos VIII y IX, bajo la influencia de la nobleza visigoda que habita el palacio de Santa María del Naranco, en las inmediaciones de Oviedo. Sigue la supremacía del leonés en el siglo $\mathrm{X}$ y primera mitad del XI, con el traslado de la corte a León y la for-

(1) Jaime Oliver Asín. Historia de la Lengua Española. 
mación del reino nuevo, cuyos monarcas se sienten cabeza de un imperio. "Hacia la segunda mitad del siglo XI, el leonés dejó de ser dialecto propenderante $\mathrm{y}$ en su lugar el castellano mantuvo desde entonces la hegemonía lingüístice" (1). Castiella, o los castillos, se convierte de condado en Reino; de "un pequeño rincón", como dice el poema de Fernán González, en el núcleo pätriótico que va extendièndose mediante sucesivas anexiones e impetuosamente dirige y culmina le empresa de la Reconquista y unidad españolas. Castilla va imponiéndose con la espada y con la lengua. La cohesión nacional se produce paralelamente a la irradiación del castellano como lenguaje común. El idioma se convierte en alta política del Estado, como en la Roma del Imperio o como entre los Incas del Tahuantinsuyo. Las ventajas de entenderse bajo un solo dictado en la norma jurídica y en la convivencia social son comprendidas como esenciales e indispensables por los conductores de este magno cometido histórico y alcanzan la plenitud de su conciencia bajo el reinado de sapiencia - a la vez política que lingüística - de los Reyes Católicos Fernando e Isabel, él, aragonés que abandona su dialecto para asumir el castellano - desde entonces español- y ella, castellana, discípula de humanistas, que propicia al gramático Antonio de Nebrijia en la tarea de "raducir y ayuntar en un solo cuerpo" lo que estaba fragmentado.

Caracteriza al castellano de taquellos siglos, tomo ya hemos dicho, su fuerza evolutiva. Esta lo eleva singularmente no solo sobre el romance mozárabe sino sobre el asturiano y leonés que le habían precedido en hegemonía y sobre los más distantes en área geográfica, el gallego-portugués y el catalán. Estos dialectos aparecen como arcaizantes junto al cambiante castellano. Entre aquellos se conservan, con más o menos vigor, peculiaridades fonéticas que éste supera después de su etapa prehistórica. Sucede así con el diptongo ai; latín cantai, portugués y leonés cartei, castellano canté; latín caldairu, leonés caldeiro, castellano caldero. Proceso análogo sigue la diptongación de o breve latina que en una primera etapa es ou y luego ve: puode, puede; tuorto, tuerto; fuoros, fueros. La primera etapa sobrevino en el asturiano occidental, mientras que en el castellano se ha afirmado la segunda (1). Observaciones análogas se puede hacer con respecto al diptongo latino au (teuro, touro, toro) con el grupo de consonante latinas

(1) Oliver Asín. Ibidem.

(I) Ramón Menéndez Pidal. Manual de Gramática Histórica Española. 9.13. 
mb (palumba, palumba, paloma); con el grupo inicial de consonante latinas pl (plcrare, plorar, llorar); con el grupo de consonantes latinas ct (lacte, leite, leche); y con numerosos fenómenos más al comparar el castellano ya con el dialecto mozárabe, ya con el asturiano, el leonés, el cjallego o el catalán (2). A través de estas comparaciones puede verse siempre la trayectoria acelerada del castellano. La lengua ha conseguido fijar un ideal acústico, de perceptibilidad, eiegancia y arrnonía y hacia él orienta su material sonoro, amoldándolo enérgica y rápidamente. Lo ha consegujdo casi por completo en el siglo XVI cuanto España se ambarga en la obra de la colonización de América.

El idioma transculturado en la Amórica hispana mantiene aquellos poderes germinalas $y$ es posible comprobarlo con motivo del vocabulario indígena. Ia refractación de una asombrosa naturaleza y de una sociedad desconocida sobre la conciencia de conquistadores y colonizadores motiva el apcrte americano a la lexicología española: nombres de animales y de plantas, peregrinas toponimias, onomástica de reyes y personajes fabulosos, apelativos de comidas, instituciones, edificios, costumbres y objetos de una múltiple realidad humana ingresan al castellano en abundancia. Cuando el castellano se pone a describir en la prosa de los Cronistas aquel maravilloso diorama o a narrar su legendario acontecer, placeracióni seoriza de aquel vocabulario. El castellano entonces djusta a estas palabras las mismas leyes fonéticas que acaban de presidir en la Edad Media española la transformación del latín peninsular en la lengua moderna. Ya Garcilaso Inca observa tales cambios y los llama "corrutelas" de los españoles. Juan de Arona, en el siglo XIX, (l) se refiere a palabras quechuas que reciben "la culta forma del idioma neo-latino". Y Pedro M. Benvenutto Murrieta, en śpoca moderna (2) afirma sagazmente: "Las viejas leyes del genio fonético español renovaron con las lenguas americanas lo que, en anteriores siglos y en disímil ambiente, hicieron con el idioma latino". El hecho no es insólito, por lo tanto, en los Cronistas sino es general en el castellano de América; pero en los Cronistas está su primer documento. Tratándose del castellano del Perú, muchas veces la trans-

(2) Oliver Asin. Ibidem.

(1) Juan de Arona. Diccionario de Peruanismos. Observaciones Generales.

(2) Pedro M. Benvenuto Murrieta. El Lenguaje Perusno. Lima, 1936. Cap. VI. 
formación es rectificada más tarde por una influencia indígena que revitaliza el sonido americano, como veremos más adelante, haciéndolo vencer a la presión castiza, pero esto es lo excepcional y nó lo comiente. Podemos decir, como principio universal, que las palabras indígenas se incorporan al español, cinéndose a las leyes del vocalis. mo y del consonantismo históricos que rigieron en la Edad Media española para la evolución del romance y que el lenguaje de los Cronistas antiguos del Perú nos muestra los primeros testimonios de esta interesante prolongación de hechos lingüísticos. Por esta causa y por otras de su contenido espiritual, la lectura de las Crónicas puede hacerse bajo la invitación amable de los alejandrinos del Arcipreste que nos sirven de epigrale. En la exposición siguiente no agotamos ni todas las leyes fonéticas ni todos los textos. Tomamos solo algunos para ilustración. Nos basta con esbozar el tema: se trata sin duda de un asunto muy rico y muy vasto que puede daf ocasión a estudios más amplios y prolijos.

Presentaremos a continuación los ejemplos más representativos. Escogemos para ello a los cronistas Pedro Pizarro, Cieza de León. Sarmiento de Gamboa, Fray Martín de Murúa, Fernando de Montesinos y Garcilaso de la Vega, pertenecientes al siglo XVI, con excepción de los dos últimos, que escribenten el XVII. Contrariamente a lo que podía pensarse, en Garcilaso no da cima sino retrocede el proceso lingüístico. Garcilaso, peruano y guuechuista representa aquella detención - retomo a lo primitivo de la fuente idiomática que también se advierte en la historia del romance medioeval. Menóndez Fidal caracteriza con el nombre de semicultismos (1) a aquellas voces que comienzan una evolución de carácter popular pero que luego la suspenden en determinado momento debido a una influencia erudita. Así saeculu debió dar sejo como especulu dió espejo; pero a causa del uso constante de la palabra latina en la predicación eclesiástica, el cambio quedó en sieglo y siglo. En Cieza León leemos Vilaoma (sumo sicerdote), que en quechua era Willakk-úmo. Garcilaso dice: "Al sumo sacerdote llaman los españoles Vilaoma, habiendo de decir Uillac Umu". En el castellano moderno ha quedado Víllac Umo, casi conforme con su corrección. El semicultismo garcilaslano, sin embargo, no triunfa siempre: decimos ahora Andes, como Pizarro y como Cieza, y no Antis como él aconseja, porquer.para reprimir la modificación castiza debieron actuar muchos otros

(1) R. Menéndez Pidal. Ibidem. 
factores expresivos y no solamente su voz lejana y literaria. Mejor dicho. su fidelidad al sonido primigenio es coincidente con cierta fidelidad popular en los casos en que aquel triunfa; y está huérfana de ella en los casos que son mas numerosos, de imposición final del molde hispánico.

Enel campo de las vocales encontramos la tendencia a conveitir $u$ tónica en o, lo que puede equipararse a la u tónica breve del latín re ducida a o en el romance: del latín bucca, en el romance boca. Aparte de la mencionada voz Vilaoma, ofrecemos otros ejemplos:

1) Quechua: tupu (medida agraria); Sarmiento de Gamboa, topo; Murúa, topo; Garcilaso, tupu; castellano actuai: topo.

2) Quechua: ussuta (sandalia); Pizarro: oxota; Cieza de León: oxota; Sarmiento de Gamboa: oxota; Garcilaso: usa usuta y oxota. dando preferencia a la forma primera. En el castellano actual es ojota aunquie en algunas regiones de América sə usa excepcionalmente, según Angel Rosemblat, ushuta, usuta y osota, lo que revela diferentes fases de la transformación.

3) Quechua: uturunku (tigre americano y serpiente atigrada); Murúa: otorongo; Garcilaso: utururcu y no otorongo "conforme a la corrutela española"; castellano actual: otorongo.

4) Quechua: kuka (arbusto de hojas masticatorias); Pizarro: coca; Cieza de León:coca; Montesinos: Coca; Garcilaso: cuca "que los españo les llaman coca"; castellano actual: coca.

5) Quechua: mamakuna (jerarquía de vestales del sol); Pizarro: mamacona; Murúa: mamacona; Garcilaso: mamacuna; castellano actual: mamacona (salvo lenguaje técnico).

La palahra Cuzco parece contradecir la regla pues proviene del quechua Kkoskko (centro, punto equidistante) y los Cronistas la españolizan cambiando la o en u; Pizarro: Cuzco; Cieza de León: Cuzco; Sarmiento de Gamboa: Cuzco "que en la lengua antigua de este valle se llama Cozco"; Murúa: Cusco; Montesinos: Cusco; Garcilaso: Cozco. El fenómeno se explica por la disimilación que consiste "en suprimir la incómoda semejanza entre dos sonidos de una palabra" (1); latín: viginte; romance: veinte; latín: auscultat; romance antiguo: ascucka; ro- 
mance moderno: escucha. La fijación de Pirí -- Perú en Perú (Cieza de León: Derú; Montesinos: Pirú) es también fenćmeno disimilante por la variedad eufónica que introduce la e, vocal fuerte, junto a la u vocal débil. La sucesión de dos débiles equivalía a la monólona repetición de una misma letra. Garcilaso sostiene que en si más remoto origen la palabra tuvo e - u y que fué "corrompida" más tarde. Asimismo la e de Perú puede justificarse por las leyes del silabismo inicial segín las cuales la $i$ se convierte en e. Latín: plicare; romance: llegar. Sobre diptongaciones y otros cambios de vocales tónicas no encontramos constantes muy precisas aunque se pueden ser̃aler aislados o formando grupos pequeños.

En las vocales átomas sobresale con intenso vigor el fenómeno de conversión de las débiles $i, u$, finales de palabra en e, 0 , respectivamente, lo que concuerda con la impronta castiza. El espariol, en efecto, sólo por influencia erudita acepta palabras como crisis o espíritu terminadas en las débiles $i, u$. En las demás se cumple la regla sin vacilaciones; latín: dixi; romance: dije; latín: protu; romance: prado. En el lenquaje de los Cronistas aparece con iqual fuerza esta norma fonética. Los siguientes son eiemplos de confusión de $i$ átona tirtal con e:

1) Quechua:Tunpis (Departamento e histórico puerto del Norte del Perú) Pizarro: Tumbez; Cieza de León: Tumbez; Garcilaso: Túmpiz. Castellano actuaBtumbez ty fumbes, con predominio de esta última ortografía.

2) Quechua: maí "vaso o plato de calabaza); Miurúa: mate; Garcilaso: mati; cástellano actual: mate.

3) Quechua: Chili (País de Sudamérica); Pizarro: Chile; Cieza de León: Chile y Chili; Garcilaso: Chili.

Los siguientes son ejemplos de sustitucićn de uátona final cor o:

1) Quechua: tarpu (hospedería, mesón); Pizarro: tambo; Cieza de León: tambo; Montesinos: tambo; Garcilaso, tampu; castellano actual: lambo.

2) Quechua: Antissuyu (Región Este) Pizarro: Ardesuyo; Cieza de León: Anciesuyo; Garcilaso: Antisuyu; Castellano actual: Antisuyo.

(1) Ramón Menéndez Pidal. Ibjidemr, 66. 
3) Quechua: Kitu (Ciudad capital del Ecuador); Pizarso: Quito; Cieza de León: Quito; Murúa: Quito; Garcilaso: Quitu; Castellano actual: Quito.

Las excepciones, en que ha primado el final de palabra indigena, son numerosas, especialmente, con $i, y$ abundan en la toponimia: Huari, Ayaviri, Guecui.

En el campo de las consonantes, comprobamos de manera abundante, en primer término; la transformación de las oclusivas sordas interiores de palabra en sonoras: $p, t, k$, en $b, d$, $g$, respectivamente, de conformidad con la clásica ley histórica que se cumple de conjunto en la palabra de origen griego apoteka que da en español bodega: y separadamente en infinidad de palabras latinas al pasar al romance. Latín: lupu; romance: lobo; latín: melu; romance: miedo; latín: securu; romance: seguro. Ejemplos:

I) Quechua: Warkapanpa (Toponimia en varias regiones del Perú): Cieza de León: Guancabamba; Garcilaso: Huancapampa (como Cochapamba, Cotapampa, Carapampa y todas las tooponimias peruanas compuestas con la voz quechua panpa, castellano pampa, (que significa llanura). Castellano actual: Huancabamba como Cochabamba, Cotabambaj Cajabamba y todas las toponimies peruanas compuestas con pampa. Esta última palabra, usada en forma simple, ha mantenido la $p$ iniciâl y la $p$ medià de si origed quechus.

2) Quechua: Kúntur (ave de rapiña); Cieza de León: cóndor; Garcilaso: cúntur; Castellano actual: cóndor.

3) Quechua: Wamanka (provincia del Ferú); Cieza de Lećn: Guamanga; Murúa: Guamanca; Garcilaso: Huamanca; Castellano actual: Huamanga.

En la evolución de estas tres consonantes, se presentan casos de semicultismos que pueden atribuirse a Garcilaso o a otras fuerzas de reflorecimiento del fonetismo aborigen. Sucede así con la voz quechua Atauwalpa que se castellaniza inicialmente en Atabaliba (con vacilaciones! y que Garcilaso corrige: Atahualpa, lo que ha primado en el castellano de hoy; con la voz quechua Intí (el sol) como primera parte de palabras compuestas. Cieza de León hace Indehuaxi hoy Intihuasi y Sarmiento de Gamboa hace Indicancha, hoy Inticancha; y con la voz 
quechua inka que se castellaniza inicialmente en inga y que Garcilaso corrige: inca. En el castellano actual se pronuncia de esta manera. Existe sin embargo el apellido Inga. Los tres ejemplo que hemos puesto se refieren sucesivamente a detención en el cambio de $p=b ; t=d$ i $\mathrm{k}=\mathrm{g}$.

Varias otros fenómenos relativos a consonantes podríamos señalar como supervivencia de leyes fonéticas medioevales; pero nos limitaremos, para concluir este trabajo, a dos de ellos: uno es el cambio de $s$ en $j ;$ y otro el cambio de $r$ en 1 . En la evolución del español encontramos el origen de $j$, leira genuinamente castellana, como proveniente de $s$ inicial y además de otras letras o grupos de letras. Latín: saponem; romance: jabón; conversión que es atribuída a los moriscos en etapa relativamente moderna. Respecto a $t$ inicial $=l$, el problema es interesante porque el caso no es común en español. Menéndez Pidal dice (1): "Ia posición inicial da a las consonantes una resistencia quizá superior a la de las vocales; todas se conservan menos la $b$, y en algunos casos le $g^{\prime \prime}$. Entre las consonantes iniciales que cambian no incluye, pues, a la $r$. En posición medial, indica: "la 1 y $r$ se truecan a menudo". (2). Latín: pallidu; romance: pardo. La explicación del fenómeno peruana estaría en que la $r$ inicial indígena es de vibración corta, como $r$ simple. Nótese que el cambio se prcduce en el Perú tanto al comienzo como abinterior de da palabia. Ejemplos:

1) Quechua: Saussa (çiudad yprovincia peruana) Pizarro: Xauxa; Cieza de León: Xauxa; Sarmiento de Gambo: Xauxa; Garcilaso: Sausa "que los españoles corrompiendo las letras llaman Xauja"; castellano actual: Jauja.

2) Qucchua: Rímakk (Asiento de la antigua Lima); Pizarro: Lima; Cieza de León: Lima; Murúa. Lima; Garcilaso: Lima, generalmente, aunque preconiza y usa, a veces, Rímac que "los españoles, corrompiendo el nombre, llaman Lima"); castellano actual: Lima. Juan de Arona dice en su Diccionario: "Lima, ligera corrupción española de Rímac como se ve en Limatambo por Rímactampu y en Lunaguaná por Runahuanac, lúcuma por rugma, en que igualmente la $r$ inicial se vuelve $l^{\prime \prime}$. (I).

JOSE JIMENEZ EORJA.

(1) Ramón Menéndez Pidal. Ibídem, 36.

(1) Ramón Menéndez Pidal. ibidem, 54.

(1) Iusn de Arona. Ibidem. Lima. 